PREPARED FOR THE U.S. DEPARTMENT OF ENERGY, UNDER CONTRACT DE-AC02-76CH03073

PPPL-3821

PPPL-3821

UC-70

Generation of Ultra-high Intensity Laser Pulses

by

N.J. Fisch and V.M. Malkin

June 2003

$\left.\stackrel{M}{M}\right|_{\substack{\text { PRInCETON PLASIMA } \\ \text { PHYSICS LABORATORY }}} ^{D}$

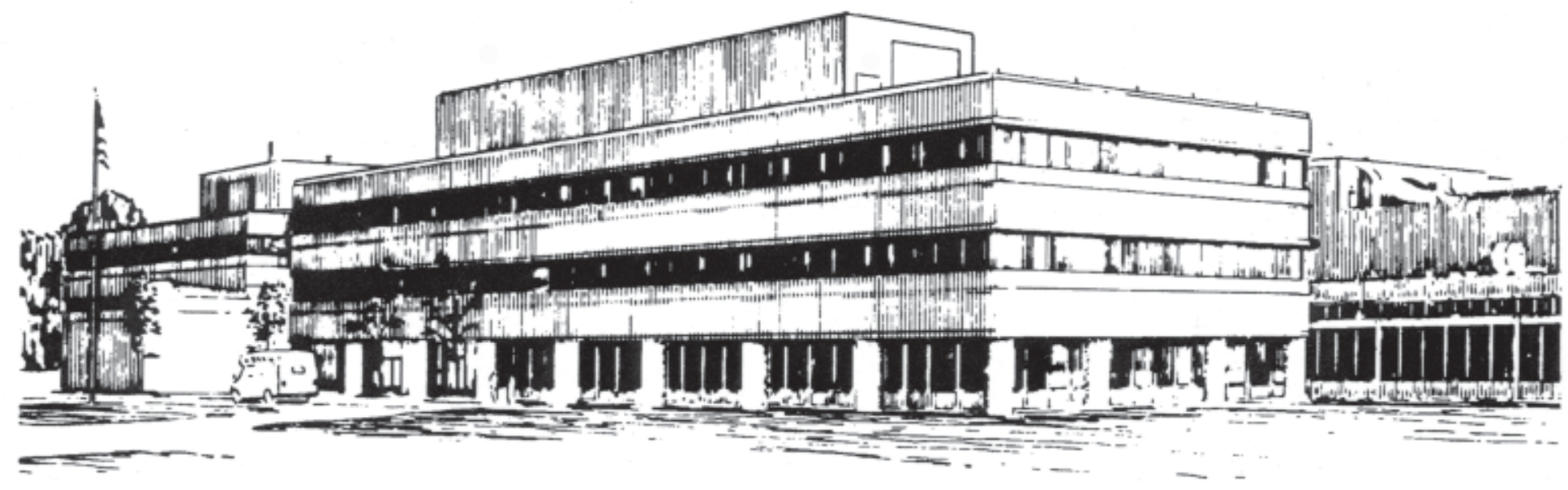

PRINCETON PLASMA PHYSICS LABORATORY PRINCETON UNIVERSITY, PRINCETON, NEW JERSEY 


\section{PPPL Reports Disclaimer}

This report was prepared as an account of work sponsored by an agency of the United States Government. Neither the United States Government nor any agency thereof, nor any of their employees, makes any warranty, express or implied, or assumes any legal liability or responsibility for the accuracy, completeness, or usefulness of any information, apparatus, product, or process disclosed, or represents that its use would not infringe privately owned rights. Reference herein to any specific commercial product, process, or service by trade name, trademark, manufacturer, or otherwise, does not necessarily constitute or imply its endorsement, recommendation, or favoring by the United States Government or any agency thereof. The views and opinions of authors expressed herein do not necessarily state or reflect those of the United States Government or any agency thereof.

\section{Availability}

This report is posted on the U.S. Department of Energy's Princeton Plasma Physics Laboratory Publications and Reports web site in Fiscal Year 2003. The home page for PPPL Reports and Publications is: http://www.pppl.gov/pub_report/

DOE and DOE Contractors can obtain copies of this report from:

U.S. Department of Energy

Office of Scientific and Technical Information

DOE Technical Information Services (DTIS)

P.O. Box 62

Oak Ridge, TN 37831

Telephone: (865) 576-8401

Fax: (865) 576-5728

Email: reports@adonis.osti.gov

This report is available to the general public from:

National Technical Information Service

U.S. Department of Commerce

5285 Port Royal Road

Springfield, VA 22161

Telephone: $1-800-553-6847$ or

(703) $605-6000$

Fax: (703) 321-8547

Internet: http://www.ntis.gov/ordering.htm 


\title{
Generation of ultrahigh intensity laser pulses ${ }^{a)}$
}

\author{
N. J. Fisch ${ }^{\text {b) }}$ and V. M. Malkin \\ Department of Astrophysical Sciences, Princeton University, Princeton, New Jersey 08544
}

(Received 18 November 2002; accepted 5 December 2002)

\begin{abstract}
Mainly due to the method of chirped pulse amplification, laser intensities have grown remarkably during recent years. However, the attaining of very much higher powers is limited by the material properties of gratings. These limitations might be overcome through the use of plasma, which is an ideal medium for processing very high power and very high total energy. A plasma can be irradiated by a long pump laser pulse, carrying significant energy, which is then quickly depleted in the plasma by a short counterpropagating pulse. This counterpropagating wave effect has already been employed in Raman amplifiers using gases or plasmas at low laser power. Of particular interest here are the new effects which enter in high power regimes. These new effects can be employed so that one high-energy optical system can be used like a flashlamp in what amounts to pumping the plasma, and a second low-power optical system can be used to extract quickly the energy from the plasma and focus it precisely. The combined system can be very compact. Thus, focused intensities more than $10^{25} \mathrm{~W} / \mathrm{cm}^{2}$ can be contemplated using existing optical elements. These intensities are several orders of magnitude higher than what is currently available through chirped pump amplifiers. (C) 2003 American Institute of Physics. [DOI: 10.1063/1.1567290]
\end{abstract}

\section{INTRODUCTION}

Laboratory laser intensities have grown remarkably during recent years due to the method of chirped pulse amplification (CPA) ${ }^{1,2}$ In CPA, optical gratings are used to chirp and stretch a short pulse. A broadband optical amplifier is then employed to amplify the chirped low-power stretched signal. Complementary gratings then reconstitute the original, but now highly amplified, signal.

This method has been extraordinarily successful, but it does require a final material grating subject to fluence limits. The method also requires uniform amplification over a broad bandwidth. ${ }^{3}$ Damage thresholds for gratings made out of fused silica for $1 \mu$ light are currently in the range of $2-4 \mathrm{~J} / \mathrm{cm}^{2}$ for pulse widths of 1 to $10 \mathrm{ps}$, and about $0.3 \mathrm{~J} / \mathrm{cm}^{2}$ in the range of $10 \mathrm{fs}{ }^{4}$ Thus, for ps pulses, unfocused power in the range of TW/ $/ \mathrm{cm}^{2}$ are achievable, with a vacuum focus then able to bear on target power intensities in the range of $10^{21} \mathrm{~W} / \mathrm{cm}^{2} .^{2}$

However, to achieve very much higher focused power intensities requires compression to much shorter times of much higher fluence pulses. The use of $10^{3} \mathrm{~cm}^{2}$ gratings withstanding fluences on the order of $1 \mathrm{~J} / \mathrm{cm}^{2}$, while feasible, is already expensive and technologically challenging. Using CPA to attain much higher power will require gratings that will eventually be too large to produce. Moreover, ultrahigh focused power in the UV will be even more difficult to achieve. At wavelengths shorter than a micron, processing high power with the current gratings technology is even more difficult if not infeasible. Whereas at one micron, fluences of several $\mathrm{J} / \mathrm{cm}^{2}$ can be contemplated, at shorter wavelengths, only much lower fluences can be imagined.

\footnotetext{
${ }^{a)}$ Paper KI1 3, Bull. Am. Phys. Soc. 47, 181 (2002).

${ }^{\mathrm{b})}$ Invited speaker.
}

At $\mathrm{TW} / \mathrm{cm}^{2}$, however, electrons in the plasma are not at all relativistic, and the plasma is hardly perturbed. The desired nonlinear interactions in the plasma may therefore be carefully controlled. The limiting effects in plasma are the nonlinear effects associated with nearly relativistic electron velocities in the wave fields; hence, at higher frequency, the plasma is even more capable of processing high power, since the velocities in a constant power laser scale inversely with frequency. Thus, plasma is ideal for applications for processing the highest possible power, and even more appropriate for short wavelength light, even as, for short wavelength light, material gratings become less appropriate.

Plasmas have, in fact, long been contemplated as media suitable for compressor/amplifiers. First observed in liquids, ${ }^{5}$ the compression of laser light through Raman backscatter has been observed also in gases and plasmas. An early review of pulse compression of excimer lasers in gases is given by Ref. 6 . The advantages of using plasma were recognized by Capjack et $a l .{ }^{7}$ Raman compression in gas mixtures, from tens of nanoseconds to tens of picoseconds, has been achieved at about $25 \%$ efficiency for energies in the range of 0.1 to $10 \mathrm{~J}^{8,9}$

In the counterpropagating geometry, the energy is stored in a long low-intensity pump pulse, or possibly a train of low-intensity pump pulses. The short counterpropagating pulse, or "pumped pulse," can achieve intensities far higher than the pump pulse, so long as it remains short. Of course, for very high powers, Raman media other than plasmas will not be practicable. The early work on compression in plasma was focused on low-power regimes, where the Raman backscattering took place in the stationary regime, namely where the plasma wave was highly collisionally damped. The issues that plagued this early work in plasma were the stringent requirements on plasma homogeneity (because of narrowbandwidth amplification) and because the amplification 
lengths were long, in part due to the collisional damping that reduced the efficiency of the interaction.

Research on the evolution of Raman instabilities has been an active topic of research in plasma for over 30 years (see, e.g., Ref. 10). However, recently, it was suggested that Raman amplification and compression in a plasma at high power commands particularly favorable effects. ${ }^{11-13}$ In the transient high-power, pump-depletion regime, which supports the so-called " $\pi$-pulse regime" solutions, a counterpropagating short pulse is resonantly amplified even as it compresses further within the plasma. The pulse is amplified rapidly, before competing deleterious effects, such as modulational or other deleterious instabilities, ${ }^{14,15}$ have a chance to develop. The plasma is terminated, in fact, just to avoid these instabilities. However, before the plasma is terminated, perhaps after $1 \mathrm{~cm}$, the short pulse can grow to extraordinary unfocused powers, with the vacuum focusing available for focusing to higher intensities yet.

Two main themes are developed here. The first theme is that much higher focused intensity than is presently contemplated by present technology might be envisioned through dividing the optical system requirements, with one optical system pumping a plasma (or, more accurately, pumping the short seed pulse), and the second optical system releasing the plasma energy in a short time scale with precision focusing. Like with other pumped laser media, the lasing technology is simplified when requirements on the pump are mainly for net energy and not for precision optics. The second theme is that in using the resonant Raman backscatter effect to compress the laser power, there are important tools available to select the desired interaction and to avoid deleterious effects. These tools involve the relative imperviousness of the desired nonlinear effect to detuning of the resonance; thus by chirping the pump, and by employing gradients in the plasma density, it is possible to selectively detune the deleterious effects even as the desired effect persists.

The paper is organized as follows. In Sec. II we show how plasma may be used to divide the optical system requirements into two optical systems, thereby creating a successor technology to CPA. In Sec. III we describe the main regimes of pulse compression, with an emphasis on the regime of resonant Raman backscatter with pump depletion. In Sec. IV, we give the basic three-wave coupled equations that describe the RBS regime. In Sec. V, we discuss competing effects and show that this regime is particularly attractive because there are tools that allow the suppressing of deleterious competing effects while selecting the desired resonant effect. In Sec. VI, we discuss the certain spinoff applications. In Sec. VII, we summarize the main conclusions and point to outstanding issues worthy of continued investigation.

\section{DUAL OPTICAL SYSTEMS}

To achieve the compression of high laser energies through conventional chirped pulse amplification methods requires a large aperture diffraction grating, both to avoid laser-induced damage as well as to avoid the nonlinearities in refractive index that occur at high optical intensities. Focusing is limited by wave-front aberrations, which are invariably more difficult to control in large-diameter optics, a difficulty aggravated by successive amplification stages and thermal fluctuations of optical components near threshold-damage limits. Although some methods ${ }^{16}$ are available to control these aberrations or to compensate for them, either statically or adaptively, ultimately, the use of larger and larger apertures, at high power, will make diffraction-limited focusing ever more difficult.

The above limits on CPA remain true notwithstanding recent improvements in chirped pulse amplification methods through nonlinear optical techniques have extended the range of frequencies, powers, intensities and pulse durations, particularly through the use of optical parametric chirped pulse amplification (OPCPA). ${ }^{17-19}$ These techniques give large gain bandwidth amplification with high beam quality, and can serve usefully as a front-end preamplifier for large-scale Nd:glass facilities. ${ }^{20}$ However, both CPA and derivative techniques like optical parametric chirped pulse amplification (OPCPA), and related extensions to shorter wavelengths yet, ${ }^{21}$ still rely upon the time-stretching, broadband amplifying, and time-recompressing of a short pulse. It remains that higher laser pulse energies can only be contemplated with larger diffraction-grating apertures, which are still subject to the limitations of both laser-induced damage and thermal fluctuations. Thus, even if these gratings could be built, diffraction-limited focusing will remain technologically difficult. The grating apertures are also necessarily even larger for very short pulses, since the tolerable fluences are even smaller for very short pulses. ${ }^{4}$

The key to much higher power, like in other lasing schemes, is to use two optical systems: one, a long-duration high-energy low-precision pump; and, two, a precision optical focusing system. Here, the first is effectively used to pump the plasma, making it an active medium, and a second one to extract the energy from the plasma, compress it in time, and focus it. The first optical system, which can be the output of a chirped pulse amplifier, or an OPCPA, delivers the energy, but need not provide a strong focus. Since it is a conventional optical system, limited to fluences on the order of $\mathrm{J} / \mathrm{cm}^{2}$, it must have a large optical aperture in order to deliver substantial energy. What we will show in the present work is that new laser opportunities arise because the optical requirements on the pumping energy, while severe in terms of power handling, are mild in terms of precision optics, such as focusability, tunability, intensity variations, or phase coherence. Since the second optical system is relieved of the power handling, it can provide more easily a strong vacuum focus. The focusing optical system has requirements neither for large apertures nor for high power. By separating the optical systems thusly, one has one system handling high power and fluence, and a second focusing system handling only low power. Since the focusing system does not operate near damage threshold limits nor is subject to thermal stresses, it can be expected that the system can be far more easily optimized for precise focusing.

To see how this occurs, we consider for illustration a specific example of compression of $1 \mu$ light, for example, from $50 \mathrm{ps}$ to $40 \mathrm{fs}$ (see Table I of Ref. 13). To accomplish this compression, consider the two optical systems depicted 


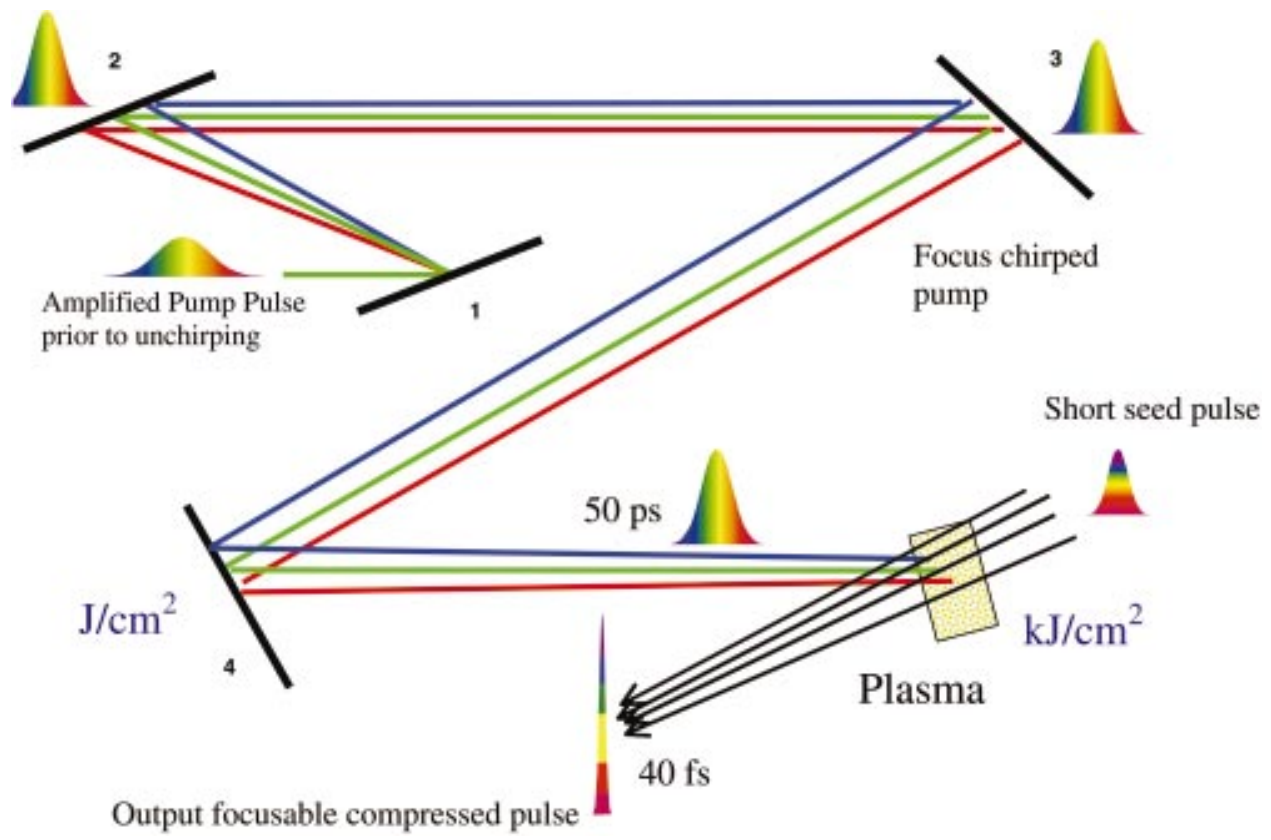

FIG. 1. (Color) Dual optical systems for RBS-CPA pulse compression. The first optical CPA system, acting as a high-energy pumping lamp, delivers fluences of $\mathrm{kJ} / \mathrm{cm}^{2}$ to the plasma. The second, low-power, optical system extracts the power by RBS, compresses it in time, and focuses it precisely on target.

in Fig. 1. In the first system, a short pulse, of say $100 \mathrm{fs}$, is sent through a stretching grating, separated into frequency components, and then sent through broadband optical amplifiers, as would be the case for the first steps of a chirped pulse amplifier. These steps are not shown, but Fig. 1 picks up the amplified chirped pulse at this point, where it is perhaps $2000 \mathrm{ps}$ long, impinging on grating 1. In a full CPA system, the pulse would be subjected to complementary gratings $\mathbf{2}, \mathbf{3}$, and $\mathbf{4}$, which provide complementary path lengths for the different frequency components in order to reconstitute as closely as possible, at an amplified intensity, the original unchirped 100 fs signal. In fact, for the purposes here, where neither full reconstitution is necessary, nor full unchirping is necessary, the complementary gratings $\mathbf{2}, \mathbf{3}$, and 4, can be used to reconstruct partially the original signal, so that a chirped pulse emerges from grating 4 at perhaps 50 ps in length. Alternatively, the precision requirements on the stretcher/compressor complementary gratings, as well as on the broadband amplifiers, may be reduced substantially, say by beginning with a short pulse of as long as 50 ps in length rather than 100 fs. In either case, from grating $\mathbf{4}$ emerges a chirped pulse of about 50 ps duration with fluence on the order of $\mathrm{J} / \mathrm{cm}^{2}$. It is important, and not too difficult, to focus beyond the plasma target, so that the plasma is irradiated by fluence on the order of $\mathrm{kJ} / \mathrm{cm}^{2}$. To do so requires a focus in linear dimension of only a factor of about 30 between the grating aperture and the plasma dimension.

The second moderate-aperature low-power optical system delivers the short, counterpropagating, focusing pulse that captures the pump $\mathrm{kJ} / \mathrm{cm}^{2}$ fluence, effectively compressing it in time, and focusing it in space. The unfocused output intensity from the plasma can be on the order of $10^{17} \mathrm{~W} / \mathrm{cm}^{2}$, and thereafter further focused in the vacuum. The key technological point of the compression optical system is that, at low power, the focusing can be precise; the key scientific point of interest is that the focusing tends to be retained through the plasma layer despite aberrations within the pump, inhomogeneities of the plasma, or small imperfections in the pulse wave-front itself, as we discuss below.

In the above example, a pump aperture of about $10 \mathrm{~m}^{2}$, possibly comprised of several individual gratings each operating at the fluence threshold of $4 \mathrm{~J} / \mathrm{cm}^{2}$, could process 400 $\mathrm{kJ}$. With a focus length of $30 \mathrm{~m}$, and placed 29 meters from a $10 \mathrm{~cm}$ by $10 \mathrm{~cm}$ plasma of depth about $0.7 \mathrm{~cm}$, this $f / 10$ grating produces fluences of $4 \mathrm{~kJ} / \mathrm{cm}^{2}$ in the plasma. Since the amplification can tolerate incoherence, separate pumps processed by different sets of gratings can be brought to focus on the same patch of plasma (or on adjacent patches of plasma) so long as the illumination is smooth. The second optical system can have an aperature of only, say, $0.5 \mathrm{~m}$, and, handling only the low power seed, can then focus $1 \mu$ light at $f / 3$ to a spot size of $10 \mu \times 10 \mu$, at 10 exawatts. The focused intensity of the resulting $40 \mathrm{fs}$ pulse is then $10^{25} \mathrm{~W} / \mathrm{cm}^{2}$. Some power is necessarily deposited in the plasma through the Langmuir wave by the Manley-Rowe relation (see the next section); for a plasma of density $7 \times 10^{18} \mathrm{~cm}^{-3}$, that turns out to be about $380 \mathrm{eV}$ per electron, which is within the allowable temperature regime, i.e., still cold enough to avoid appreciable Landau damping (see, e.g., Ref. 22).

Just for example, suppose instead that a 10 fs output pulse were required. This would be more difficult, but not necessarily impossible, by the current technique. Since the output pulse length goes as $a_{0}^{-2 / 3},{ }^{13}$ to compress to $10 \mathrm{fs}$, with powers and power densities 4 times higher, requires compressing the CPA output pulse a factor of 64 to about 625 $\mathrm{fs}$, and then employing a plasma of only about $0.1 \mathrm{~mm}$ thickness. This calculation assumes also that the pump fluence is unchanged. Note, however, that at $625 \mathrm{fs}$, the fluence limit of the pump might be as much as halved over what it might be at $40 \mathrm{ps}$, in which case to achieve $4 \times 10^{25} \mathrm{~W} / \mathrm{cm}^{2}$ would require twice the pump aperature. A more serious limitation arises from the fact that the heat is deposited in only $1 / 64$ of the number of electrons, which is over $20 \mathrm{KeV} /$ electron. If 
it were dissipated within the seed pulse, the retained heat would produce a plasma with a temperature so high that the Langmuir wave would be damped, which would inhibit the laser coupling. However, this energy dissipates behind the amplified pulse, so that the plasma heating ahead of the pulse may still be tolerable. Thus, while the full scenario for compression at $1 \mu$ to $10 \mathrm{fs}$ remains to be worked out in detail, and is clearly more difficult than to $40 \mathrm{fs}$, it can still be envisioned.

It would be natural to compress to $10 \mathrm{fs}$, however, at shorter wavelength, say $1 / 4 \mu$. In this case, a $12.5 \mathrm{ps}$ pump results in a $10 \mathrm{fs}$ output pulse at fluence of $16 \mathrm{~kJ} / \mathrm{cm}^{2}$, after traversing $0.18 \mathrm{~cm}$ of plasma at density of $1.1 \times 10^{20} \mathrm{~cm}^{-3}$. In this case only $400 \mathrm{eV}$ is deposited per electron, well within the tolerable heating range. Of course, it is possible to arrange at $1 / 4 \mu$ both for longer pump and longer plasma.

What these examples tell us is that, while the complete scenarios in some cases remain to be worked out in detail, there appear to be self-consistent operating regimes with extraordinary compression properties. The system needed for compressing and focusing laser pulses to these huge powers and intensities can be much more compact than what can be achieved by CPA devices alone. Note that the optical system for producing the pump pulse is an independent element. Therefore, as new advances in laser technology appear, such as through CPA techniques, they can be utilized in the present scheme as pump beams, thereby to achieve an even more compact amplifier addressing even higher technological limits.

\section{REGIMES OF PULSE COMPRESSION}

Although we focus here on the resonant transient Raman backscatter regime, at very high power, there are in fact several mechanisms by which the pump power might be coupled into the counterpropagating pulse (see, e.g., Ref. 23), in addition to the regimes of low-power pulse compression, where the plasma wave tends to be heavily damped. One coupling mechanism at high power involves a so-called "superradiant" or Compton scattering, where the nonlinear interaction of the plasma electrons with the lasers dominates the plasma restoring motion due to charge imbalance. ${ }^{24}$ In this regime, the pump laser is of somewhat higher frequency than the pumped laser; the pulse length of the pumped pulse is less than a plasma length, $\mathrm{c} / \omega_{p}$; and the laser intensities satisfy $a b>\omega_{p}^{2} / 4 \omega^{2}$, where $a$ and $b$ are the pump and pumped pulse normalized vector potentials. An alternative coupling mechanism involves coupling at an ionization front. ${ }^{25,26}$ For ionization front coupling, the pump must be too weak to ionize a neutral gas, but strong enough to replenish a counterpropagating intense pulse which does ionize the gas. These conditions can be met in hydrogen or helium.

In the Raman scattering regime, where resonance is required, it can be shown that the pulse grows so fast that it can outrun the deleterious instabilities. As shown in Ref. 11, within several growth times of the deleterious instabilities, the pulse grows to amplitudes far exceeding the instability thresholds, i.e., to overcritical powers. Pump depletion quickly ensues, so that the efficiencies are limited in prin- ciple only by the so called "Manley-Rowe" relations; in other words, since a pump photon is converted to a counterpropagating pulse photon downshifted by the plasma frequency, the fraction of pump energy that will be left in the plasma wave is $\omega_{p} / \omega$. Typically, this fraction would be about $1 / 10$. The remaining $90 \%$ of the pump power can, in principle, be converted entirely to the backscattered short pulse. The pump length is optimally twice the plasma length, with the pump front leaving the plasma just as the seed pulse enters the plasma, and with the pump tail entering the plasma just as the amplified seed leaves the plasma slab. Thus, as in our example of Fig. 1, compressing a 50 ps pump requires a plasma length of about $1 \mathrm{~cm}$. In the case of resonant Raman backscattering, with say $\omega_{p} / \omega$ about 0.1 , the plasma electron density must be about $10^{19} \mathrm{~cm}^{-3}$.

\section{THREE-WAVE COUPLING EQUATIONS}

Pulse compression via the resonant Raman backscattering effect is both well-studied and inherently one of the simplest plasma effects. The output pulse reaches very high intensities, but only in the sense that it leads to extreme pulse intensities on target. However, within the plasma, the nonfocused power is not particularly high, in the sense that the plasma effects are fundamentally not extreme. The coupling occurs between essentially low-intensity counterpropagating light waves via essentially low-amplitude longitudinal cold plasma oscillations. The motion of electrons in the cold plasma wave is coherent, essentially sinusoidal motion, so long as the plasma is suffiently dense to avoid wave breaking. The motion of electrons in the lasers are also nonrelativistic. Only as the pulse is amplified to its maximum value, which is essentially at the point that these effects are no longer so simple, do the electron trajectories begin to become complicated. At that point, other effects indeed enter, and that is roughly the boundary of the regime which can be considered to be the resonant Raman backscattering regime. And at that point the plasma is terminated and the pulse is extracted.

Hence, in the resonant Raman backscattering regime, there should be only a limited number of surprises in our understanding of the plasma effects. The short time scales involved mean that only electron dynamics, rather than ion dynamics, enter importantly. This further limits the possibilities of unintended effects that could hinder the useful effects. Yet in this regime the time scales are not so short that the waves cannot be simply described as envelope pulses with a central carrier frequency. In compressing from tens of picoseconds to tens of femtoseconds, there will still be many laser wavelengths in the pulse wave packet. Thus, there should be high confidence in writing the resonant Raman backscatter equations as

$$
\begin{aligned}
& a_{t}+c a_{z}=\omega_{p} f b, \\
& b_{t}-c b_{z}=-\omega_{p} f^{*} a, \\
& f_{t}=-\omega a b^{*} / 2 .
\end{aligned}
$$

Here $a$ and $b$ are vector-potential envelopes of the pump and pulse, respectively, in units of $m_{e} c^{2} / e \approx 5 \times 10^{5} \mathrm{~V}$, and 
$f$ is the envelope of the Langmuir wave electrostatic field $\vec{E}=E \vec{e}_{z}$ in units of $m_{e} c \omega_{p} / e=c \sqrt{4 \pi m_{e} n_{e}}$ $\approx \sqrt{n_{e}\left[\mathrm{~cm}^{-3}\right]} \mathrm{V} / \mathrm{cm}$, defined by formulas

$$
\begin{aligned}
& \left(A_{x}+i A_{y}\right) e / m_{e} c^{2}=a e^{i\left(k_{a} z-\omega_{a} t\right)}+b e^{i\left(k_{b} z-\omega_{b} t\right)}, \\
& \text { Ee/m } m_{e} c \omega_{p}=f e^{i\left[\left(k_{a}-k_{b}\right) z-\omega_{p} t\right]}+\text { c.c., }
\end{aligned}
$$

where $A_{x}$ and $A_{y}$ are components of the real vector-potential $\vec{A}$ in the plane transverse to the propagation direction $z$; for the pump propagating in the positive and the seed-pulse in the negative direction, $k_{a}=\sqrt{\omega_{a}^{2}-\omega_{p}^{2}} / c$ and $k_{b}$ $=-\sqrt{\omega_{b}^{2}-\omega_{p}^{2}} / c ; \omega_{p}, \omega_{b}$, and $\omega_{a}$ are the plasma, laser-seed and laser-pump frequencies, and subscripts $t$ and $z$ denote time and space derivatives. The pulse duration is larger than $\omega_{p}^{-1}$. Both lasers are circularly polarized. Self-nonlinearities of lasers and Langmuir wave are neglected. Plasma ions are assumed to be immobile. The Langmuir wave group velocity is neglected in comparison with the speed of light. For $\omega_{b}$ $\gg \omega_{p}$, one may assume, as it is done above, $\omega_{a} \approx \omega_{b}=\omega$ and $k_{a} \approx-k_{b} \approx \omega / c$ in all the equation coefficients.

An initially weak seed $b$ will be amplified by an undepleted pump field; in the constant pump or linear regime, exact solutions exist which show that while the seed front moves with the velocity of light $c$, the envelope maximum only moves with $c / 2 .{ }^{27}$ Hence, in this regime the counterpropagating wave is stretched. It is only in the nonlinear regime, i.e., the pump-depletion regime, that the pulse is compressed. In this regime, the pulse front effectively shadows the pulse maximum and tail, so that the maximum catches up to the front. Also, in this regime, all short enough and intense enough initial seeds will asymptotically reach the $\pi$-pulse solution. ${ }^{11}$ That a self-similar solution, depending only on the ratio $z / t$, exists can be seen from a scaling of the equations. Essentially, the Raman depletion of the pump by the seed takes place in a distance that varies inversely with the seed pulse amplitude. This is also the effective width of the seed pulse, since what is further than this distance behind the seed front is effectively shadowed. However, at complete pump depletion, the pulse energy must grow linearly with distance (or time) traveled, because that is all the available energy in the pump. Thus, the amplitude and energy of the seed pulse must grow linearly with distance, while the width contracts inversely with distance. Thus we expect a selfcontracting self-similar asymptotic solution. The $\pi$-pulse solution has about $53 \%$ of the energy in the first lobe, and the rest of the pulse energy appears in succeeding lobes. If there is a process that disrupts the Langmuir wave, say Langmuir wave breaking or collisional damping of the Langmuir wave, then as much as $80 \%$ of the pulse energy can be captured in the first lobe, since the regeneration of the pump will be incomplete.

\section{SELECTIVE DETUNING AND AVERAGING}

It remains important that the amplified seed power can be focused on target by retaining the integrity of the seed pulse phase fronts even as plasma inhomogeneities are encountered. Equally important issue is the ability of the pump to traverse the full plasma length without undergoing prema- ture Raman backscattering off plasma noise, prior to encountering the pulse. It turns out that both the focusing of the pulse and the stability of the pump and pulse to deleterious instabilities can be helped by favorable detuning effects and favorable averaging effects.

The fact that, in the nonlinear regime of the resonant Raman backscatter interaction, the seed pulse shortens as it is amplified has important implications. Because the bandwidth of the seed is growing, this desired interaction is less susceptible to detuning of the resonance than would be resonant backscatter interactions growing up from noise in the linear regime. $^{12}$

This suggests that a number of unwanted resonant effects might be avoided through detuning. There are essentially two tools for detuning: One is to change the pump frequency, such as by introducing a frequency chirp to the pump, with the effect that any wave traveling through the pump, such as Raman backscatter from noise, sees a different pump frequency as it is convectively amplified, but eventually is tuned out of resonance. The second tool for detuning is to change the plasma frequency, such as by introducing a density gradient, which means that the plasma frequency changes axially. Thus, forward-scattered light co-propagating with the intense pumped pulse would be born in resonance with the pumped pulse, but as both pulse and scattered signal co-propagate some distance, the plasma no longer accomodates a resonant interaction between the two waves.

In fact, detuning ideas were recognized for curing similar effects in gas-based Raman compressors. The Raman medium could be made to have a Raman gradient, where the Raman frequency would change with axial position. Moreover, it was also recognized, ${ }^{28}$ that the pump entering the plasma could be chirped too. In plasma, the issues are somewhat different, because backward scattering tends to be more important than forward scattering, and, more importantly, because the broad bandwidth pulse is relatively immune to detuning.

Thus, in a plasma, in the resonant Raman backscatter regime, suitable detuning can selectively mitigate a number of potential problems: First, detuning can stabilize the pump as it traverses the full plasma before it encounters the seed pulse, where it may be subject to Raman backscatter arising from noise. ${ }^{12}$ Second, detuning can stabilize forwardscattering instabilities of the pumped pulse ${ }^{29}$ note that although forward-scattering tends to be small in a plasma, the seed pulse grows to intensities much larger than the pulse and Raman forward-scattered waves can co-propagate with it. Third, in addition to the $\pi$-pulse solution, there is also the possibility of $2 \pi$-pulse solutions. These solutions to Eqs. (1)-(3) arise when the pulse front is not abrupt enough. They are deleterious, representing superluminous precursors growing ahead of the pulse, that deplete the pump and spoil the plasma ahead of the pulse. However, since they require a precise resonance, they may also be selectively extinguished through detuning. ${ }^{30}$

Random density inhomogeneities may defocus the pumped pulse, but the pulse tends to average over the density fluctuations if there are many correlation lengths within the plasma. ${ }^{31}$ Because of this averaging, only some of the signal 
is deflected, and the phase front of the majority of the signal remains intact and focusable. Moreover, the effect of the inhomogeneities is most pronounced in the linear regime, where the resonance bandwidth is narrow; thus, as for the other detuning effects, the deleterious instabilities arising from noise, such as the premature backscattering by thermal noise of the pump, tend to be selectively mitigated by density fluctuations, while the broadband desired output signal is relatively immune. ${ }^{31}$

This averaging is also important in that variability in the pump also tends to be averaged over. It was recognized early $\mathrm{on}^{5}$ that one of the advantages of the backscattering geometry is that intensity variations in the pump do not detract from the output seed quality, because of the averaging of the effect of the pump amplitude fluctuations. Moreover, pump phase fluctuations are also accommodated, because the seed front, encountering fresh plasma as it propagates into the pump, generates a plasma wave of whatever phase is necessary to deplete the pump, while the seed phase itself need not shift. The robustness of the phase fronts to a variety of perturbations in the seed and the pump was confirmed by Fraiman et al. in two-dimensional numerical simulations. ${ }^{32}$ Similarly, even the simultaneous use of pumps with different frequencies not only can access favorable depletion regimers, but does so with robustness to density fluctuations. ${ }^{33}$

The implication of this robustness to the pump quality is that the division into two optical systems discussed above becomes even more practical. If temporal coherence of the pump is not critical, then many pump lasers can be focused onto the plasma, without the need to create one very large, highly coherent, pump laser. Thus, in addition to effecting the temporal compression of the pump power delivered over longer times, the well-focusable seed pulse acts to focus the pump power, that by itself, because of incoherencies, would be far from diffraction limited. Incoherent pumps, in fact, produce robust compression, much in the same way as random phase lasers avoid instabilities in pellet compression. ${ }^{34}$

\section{VARIATIONS AND SPIN-OFF APPLICATIONS}

The compression effect is likely best achieved through the careful preparation, alignment, and timing of a counterpropagating seed pulse. However, more advantageous scenarios might also be imagined. For example, it would be to great advantage if the timing could be achieved automatically, i.e., if the backscattered wave arose spontaneously at just the right spot and time in the plasma, namely just at the point of the pump front as it is about to exit plasma. In principle, backscattering off of stationary plasma oscillations near the plasma exit could accomplish this. The seed is then the plasma oscillation, which has nearly vanishing group velocity, and hence does not need to be timed precisely. The backscattered pulse is then generated by the same resonant backward Raman scattering process off of a prepared plasma wave. While this method, in principle, is the most simple to implement, it is an open question whether the simplified method can result in a backward-propagating seed signal with the correct parameters to enter into a useful compression regime.
Another variation might be to implement the counterpropagating waves in a plasma channel, or in a wave guide, where the effects of the wave guide walls can alter the dispersive properties of the light. ${ }^{35}$

The counterpropagating very high-power wave geometry might also have some interesting spin-off applications. One possibility is for the purpose of accelerating relativistic particle beams through the plasma wave produced in the counterpropagating geometry. There may be advantages, similar to the dual optical system for pulse compression, in employing a long pump pulse to provide the main power, while a short counterpropagating probe can produce a precisely aligned plasma wake. ${ }^{36}$ There is also the possibility of enhancing the growth rate of the plasma wave, through a related four-wave interaction in the counterpropagating geometry. ${ }^{37}$

A second example is for processing optical information at high power. ${ }^{38}$ Note that in employing stimulated Raman backscattering in a plasma, after the two counterpropagating light waves pass through the plasma, there remains the nonpropagating plasma wave for at least a few collision times. This plasma wave carries information about the laser pulses. Consider the pump wave to carry information through amplitude modulation. Now use a short counterpropagating probe wave at the Raman downshifted frequency. The plasma wave will then capture the amplitude variations of the pump wave. If the plasma is then probed with a short laser pulse, the information stored in the plasma wave can be retrieved in a second scattered electromagnetic wave. In addition to simply retrieving it at a later time, the optical information captured in the slow moving plasma wave might be otherwise processed by being probed with more complicated reference beams. Thus, certain operations might be performed on the slower time scale, such as taking the derivative of the pulse form or performing other simple mathematical operations. Suppose further that either the recording or retrieval pulses have transverse phase variation, such as focussing or defocusing wave fronts. The transverse phase advances can then be captured either in the plasma wave or in the retrieval process. The result is that the original data pulse upon retrieval can be brought to focus or magnified.

The retrieval of optical information might also be used for pulse shaping at high frequency. Quantum control by lasers relies upon detailed pulse shaping techniques; ${ }^{39}$ although well developed at optical wavelengths, these techniques are lacking in the VUV and even in the UV. By capturing a pulse in a plasma wave, there is the opportunity to deform it along the lines outlined for other processing, before reconstituting the EM wave. It would be important to examine whether plasma can offer opportunities either at short wavelength (UV) or at high power where conventional techniques, where even new Raman techniques, ${ }^{40}$ may not deliver. What is important as well is that even if these techniques fail to produce new pulse-forming technology, the probing of the Raman excite plasma wave is itself very important information in validating the underlying plasma physics.

Another example of spin-off technology is the use of the very high power laser intensities contemplated here to ex- 
plore new regimes for fast ignition. ${ }^{41}$ Higher laser power might lead to higher energy electrons delivering the required energy to the pellet core at smaller currents to achieve the pellet ignition. ${ }^{42}$

\section{CONCLUSIONS}

Plasma is the natural medium for processing high power and high fluence lasers. There are, in fact, several backscatter effects, in somewhat different regimes, that may lead to compression of lasers at high power in plasma. However, particularly attractive is the regime of resonant Raman backscatter, in the pump-depletion regime, which supports $\pi$-pulse selfsimilar self-contracting solutions.

It is important to note that this is an essentially simple effect in the sense that electron motion is quite coherent even as high power pulse compression is achieved. Significantly, since the effect relies on resonance, it is possible to introduce detuning in such a way that the amplification of unwanted noise can be suppressed even as the useful amplification persists.

Among these issues that may be addressed by selective detuning is the stability of the pump to deleterious instabilities, the unwanted appreance of superluminous precursors, and the stability of the pulse to deleterious instabilities as well. The robustness of the self-contracting broadband pulse to detuning also makes it less sensitive to random plasma inhomogeneities than are certain unwanted resonant interactions. Equally important, is that the pulse tends to retain its focus as it averages over phase imperfections and intensity variations in the pump pulse.

It should be recognized that while there have been many related experiments, none have realized compression at high power. Recently, backscattered amplification was reported in a Li-F recombining plasma, ${ }^{43}$ as well as in copper microcapillaries. ${ }^{44}$ Resonant amplification appears to be observed as well in recent gas jet experiments. ${ }^{45,46}$ However, a $\pi$-pulse solution, or even any compression solution, has yet to be demonstrated in plasma.

Yet the compression of lasers in plasma is likely to be a key component to the eventual realization of much higher laser intensities than are presently achieved. As applications emerge particularly for submicron wavelengths, the compression effect in plasmas will become even more central to the development of suitable high-power lasers, since alternative means of pulse compression are not suited to short wavelength.

The successor technology to chirped pulse amplification envisioned here employs two optical systems: one, likely the output of conventional CPA technology today but with reduced fluence requirements, or, more advanced technology as it it becomes available, simply pumps energy into the plasma, much as a flashlamp, to make the plasma active. The second optical system provides precise optics at low power so that a focusable seed can extract this energy in a short time, with likely more precise focusing than would be possible with the high power pump. The possibilities of this dual system allow us to contemplate very compact systems producing exawatts of $1 \mu$ light focused onto targets of just sev- eral wavelengths, leading to intensities higher than present technology can offer by several orders of magnitude.

\section{ACKNOWLEDGMENTS}

The authors are particularly in debt to G. Shvets for discussions and joint efforts on the early work on methods of pulse compression in plasma. Useful discussions are acknowledged as well with S. Suckewer and J. S. Wurtele on the implementations discussed here. The authors are grateful also to D. Berger, D. Clark, I. Dodin, G. Fraiman, R. Kirkwood, A. B. Langdon, J. Meyer-ter-vehn, Y. Ping, A. Pukhov, A. Solodov, Yu. Tsidulko, E. Valeo, and N. Yampolsky for valuable collaborations and discussions.

The work is supported by Department of Energy Contract No. DE-FG030-98DP00210 and by DARPA.

${ }^{1}$ D. Strickland and G. Mourou, Opt. Commun. 56, 219 (1985); G. A. Mourou, C. P. J. Barty, and M. D. Perry, Phys. Today 51 (1), 22 (1998).

${ }^{2}$ M. D. Perry, D. Pennington, B. C. Stuart et al., Opt. Lett. 24, 160 (1999). ${ }^{3}$ G. Cheriaux and J. P. Chambaret, Meas. Sci. Technol. 12, 1769 (2001).

${ }^{4}$ B. C. Stuart, M. D. Feit, S. Herman et al., J. Opt. Soc. Am. B 13, 459 (1996).

${ }^{5}$ M. Maier, W. Kaiser, and J. A. Giordmaine, Phys. Rev. Lett. 17, 1275 (1966); Phys. Rev. 177, 580 (1969).

${ }^{6}$ J. R. Murray, J. Goldhar, D. Eimerl, and A. Szoke, IEEE J. Quantum Electron. QE-15, 342 (1979).

${ }^{7}$ C. E. Capjack, C. R. James, and J. N. McMullin, J. Appl. Phys. 53, 4046 (1982).

${ }^{8}$ H. Nishioka, K. Kimura, K. Ueda, and H. Takuma, IEEE J. Quantum Electron. 29, 2251 (1993).

${ }^{9}$ E. Takahashi, Y. Matsumoto, I. Matsushima, I. Okuda, Y. Owadano, and K. Kuwahara, Fusion Eng. Des. 44, 133 (1999).

${ }^{10}$ W. Kruer, Physics of Laser-Plasma Interactions, Frontiers in Physics Vol. 73 (Addison-Wesley, New York, 1988); M. N. Rosenbluth, Phys. Rev. Lett. 29, 565 (1972); 31, 1190 (1973); C. S. Liu, M. N. Rosenbluth, and R. B. White, ibid. 31, 697 (1973); J. F. Drake and Y. C. Lee, ibid. 31, 1197 (1973); D. W. Forslund, J. M. Kindel, and E. L. Lindman, ibid. 30, 739 (1973); P. Koch and J. Albritton, ibid. 34, 1616 (1975); R. G. Watt, R. D. Brooks, and Z. A. Pietrzyk, ibid. 41, 170 (1978); K. Estabrook, W. L. Kruer, and B. F. Lasinski, ibid. 45, 1399 (1980); K. Tanaka, L. M. Goldman, W. Seka, M. C. Richardson, J. M. Soures, and E. A. Williams, ibid. 48, 1179 (1982); A. A. Offenberger, R. Fedosejevs, W. Tighe, and W. Rozmus, ibid. 49, 371 (1982); K. Estabrook and W. L. Kruer, ibid. 53, 465 (1984); M. Salimullah, Y. G. Liu, and M. G. Haines, Phys. Rev. A 30, 3235 (1984); D. W. Forslund, J. M. Kindel, W. B. Mori, C. Joshi, and J. M. Dawson, Phys. Rev. Lett. 54, 558 (1985); H. C. Barr, T. J. M. Boyd, and G. A. Coutts, ibid. 56, 2256 (1986); C. Labaune, H. A. Baldis, S. D. Baton, D. Pesme, and T. Jalinaud, ibid. 69, 285 (1992); C. B. Darrow, C. Coverdale, M. D. Perry, W. B. Mori, C. Clayton, K. Marsh, and C. Joshi, ibid. 69, 442 (1992); T. Kolber, W. Rozmus, V. T. Tikhonchuk, Ph. Mounaix, and D. Pesme, ibid. 70, 1810 (1993); P. Sprangle, E. Esarey, and A. Ting, ibid. 64, 2011 (1990); B. Bezzerides, D. F. DuBois, and H. A. Rose, ibid. 70, 2569 (1993); P. Monaix, D. Pesme, W. Rosmus, and M. Casnova, Phys. Fluids B 5, 3304 (1993); C. Rousseaux, G. Malka, J. L. Miquel, F. Aminranoff, S. D. Baton, and Ph. Mounaix, Phys. Rev. Lett. 74, 4655 (1995); S. C. Wilks, W. L. Kruer, E. A. Williams, P. Amendt, and D. C. Eder, Phys. Plasmas 2, 274 (1995); M. J. Everett, A. Lal, D. Gordon, K. Wharton, C. E. Clayton, W. B. Mori, and C. Joshi, Phys. Rev. Lett. 74, 1355 (1995); B. B. Afeyan and E. A. Williams, ibid. 75, 4218 (1995); G. Shvets, J. S. Wurtele, and B. A. Shadwick, Phys. Plasmas 4, 1872 (1997); M. M. Skoric, M. S. Jovanovic, and M. R. Rajkovic, Phys. Rev. E 53, 4056 (1996); B. F. Lasinski, A. B. Langdon, S. P. Hatchett, M. H. Key, and M. Tabak, Phys. Plasmas 6, 2041 (1999); D. S. Montgomery, B. B. Afeyan, J. A. Cobble et al., ibid. 5, 1973 (1998); J. C. Fernandez, J. A. Cobble, D. S. Montgomery, M. D. Wilke, and B. B. Afeyan, ibid. 7, 3743 (2000); J. D. Moody, B. J. MacGowan, J. E. Rothenberg et al., Phys. Rev. Lett. 86, 2810 (2001); H. X. Vu, D. F. Dubois, and B. Bezzerides, Phys. Plasmas 9, 1745 (2002); 5, 4337 (1998); D. F. DuBois, B. Bezzerides, and H. A. Rose, Phys. Fluids B 4, 241 (1992); V. T. Tikhonchuk, J. Fuchs, C. 
Labaune, S. Depierreux, S. Hller, J. Myatt, and H. A. Baldis, Phys. Plasmas 8, 1636 (2001); H. A. Rose and D. A. Russell, ibid. 8, 4784 (2001); R. K. Kirkwood, J. D. Moody, A. B. Langdon et al., Phys. Rev. Lett. 89, 215003 (2002)

${ }^{11}$ V. M. Malkin, G. Shvets, and N. J. Fisch, Phys. Rev. Lett. 82, 4448 (1999).

${ }^{12}$ V. M. Malkin, G. Shvets, and N. J. Fisch, Phys. Rev. Lett. 84, 1208 (2000).

${ }^{13}$ V. M. Malkin, G. Shvets, and N. J. Fisch, Phys. Plasmas 7, 2232 (2000).

${ }^{14}$ A. G. Litvak, Zh. Éksp. Teor. Fiz. 57, 629 (1969) [Sov. Phys. JETP 30, 344 (1970)].

${ }^{15}$ C. Max, J. Arons, and A. B. Langdon, Phys. Rev. Lett. 33, 209 (1974).

${ }^{16}$ C. Hernandez-Gomez, J. L. Collier, S. J. Hawkes, C. N. Danson, C. B. Edwards, D. A. Pepler, I. N. Ross, and T. B. Winstone, Appl. Opt. 39, 1954 (2000).

${ }^{17}$ A. Dubietis, G. Jonusauskas, and A. Piskarskas, Opt. Commun. 88, 437 (1992).

${ }^{18}$ I. N. Ross, P. Matousek, M. Towrie, A. J. Langley, and J. L. Collier, Opt. Commun. 144, 125 (1997).

${ }^{19}$ I. N. Ross, J. L. Collier, P. Matousek, C. N. Danson, D. Neely, R. M. Allott, D. A. Pepler, C. Hernandez-Gomez, and K. Osvay, Appl. Opt. 39, $2422(2000)$

${ }^{20}$ J. Collier, C. Hernandez-Gomez, I. N. Ross, P. Matousek, C. N. Danson, and J. Walczak, Appl. Opt. 38, 7486 (1999).

${ }^{21}$ K. Osvay, G. Kurdi, J. Klebniczki, M. Csatari, I. N. Ross et al., Appl. Phys. B: Lasers Opt. B74, S163 (2002); K. Osvay, G. Kurdi, J. Klebniczki, M. Csatari, and I. N. Ross, Appl. Phys. Lett. 80, 1704 (2002).

${ }^{22}$ D. S. Clark and N. J. Fisch, "Operating regime for a backward Raman laser amplifier in preformed plasma," Phys. Plasmas (submitted).

${ }^{23}$ N. J. Fisch, V. M. Malkin, and G. Shvets, in Proceedings of the Second International Conference on Superstrong Fields in Plasma, AIP Conf. Proc. Vol. 611, edited by M. Lontano, G. Mourou, O. Svelto, and T. Tajima (AIP, Melville, NY, 2002), p. 409.

${ }^{24}$ G. Shvets, N. J. Fisch, A. Pukhov, and J. Meyer-ter-Vehn, Phys. Rev. Lett. 81, 4879 (1998).

${ }^{25}$ V. M. Malkin and N. J. Fisch, Phys. Plasmas 8, 4698 (2001).

${ }^{26}$ D. S. Clark and N. J. Fisch, Phys. Plasmas 9, 2772 (2002).

${ }^{27}$ D. L. Bobroff and H. A. Haus, J. Appl. Phys. 38, 390 (1967).
${ }^{28}$ J. A. Caird, IEEE J. Quantum Electron. QE-16, 489 (1980).

${ }^{29}$ V. M. Malkin, Yu. A. Tsidulko, and N. J. Fisch, Phys. Rev. Lett. 85, 4068 (2000).

${ }^{30}$ Yu. A. Tsidulko, V. M. Malkin, and N. J. Fisch, Phys. Rev. Lett. 88, 235004 (2002)

${ }^{31}$ A. Solodov, V. M. Malkin, and N. J. Fisch, "Random density inhomogeneities and focusability of the output pulses for plasma-based powerful backward Raman amplifiers," Phys. Plasmas (submitted).

${ }^{32}$ G. M. Fraiman, N. A. Yampolsky, V. M. Malkin, and N. J. Fisch, Phys. Plasmas 9, 3617 (2002).

${ }^{33} \mathrm{~A}$. Balakin (private communication).

${ }^{34}$ S. P. Obenschain, C. J. Pawley, A. N. Mostovych, J. A. Stamper, J. H. Gardner, A. J. Schmitt, and S. E. Bodner, Phys. Rev. Lett. 62, 768 (1989).

${ }^{35}$ P. Mardahl, H. J. Lee, G. Penn, J. S. Wurtele, and N. J. Fisch, Phys. Lett. A 296, 109 (2002); I. Y. Dodin, G. M. Fraiman, V. M. Malkin, and N. J. Fisch, J. Exp. Theor. Phys. 95, 625 (2002).

${ }^{36}$ G. Shvets, N. J. Fisch, A. Pukhov, and J. Meyer-ter-Vehn, Phys. Rev. E 60, 2218 (1999).

${ }^{37}$ G. Shvets and N. J. Fisch, Phys. Rev. Lett. 86, 3328 (2001).

${ }^{38}$ I. Y. Dodin and N. J. Fisch, Phys. Rev. Lett. 88, 165001 (2002); Opt. Commun. 214, 83 (2002)

${ }^{39}$ W. S. Warren, H. Rabitz, and M. Dahleh, Science 259, 1581 (1993); P. Brumer and M. Shapiro, Annu. Rev. Phys. Chem. 43, 257 (1992); R. Bartels, S. Backus, E. Zeek, L. Misoguti, G. Vdovin, I. P. Christov, M. M. Murnane, and H. C. Kapteyn, Nature (London) 46, 164 (2000).

${ }^{40}$ S. E. Harris and A. V. Sokolov, Phys. Rev. A 55, R4019 (1997); F. L. Kien, K. Hakuta, and A. V. Sokolov, ibid. 66, 023813 (2002).

${ }^{41}$ M. Tabak, J. Hammer, M. E. Glinsky et al., Phys. Plasmas 1, 1626 (1994).

${ }^{42}$ V. M. Malkin and N. J. Fisch, Phys. Rev. Lett. 89, 125004 (2002).

${ }^{43}$ Y. Ping, I. Geltner, N. J. Fisch, G. Shvets, and S. Suckewer, Phys. Rev. E 62, R4532 (2000)

${ }^{44}$ Y. Ping, I. Geltner, A. Morozov, N. J. Fisch, and S. Suckewer, Phys. Rev. E 66, 046401 (2002).

${ }^{45}$ Y. Ping, I. Geltner, and S. Suckewer, Phys. Rev. E 67, 016401 (2003).

${ }^{46} \mathrm{R}$. Kirkwood (private communication). 


\section{External Distribution}

Plasma Research Laboratory, Australian National University, Australia

Professor I.R. Jones, Flinders University, Australia

Professor João Canalle, Instituto de Fisica DEQ/IF - UERJ, Brazil

Mr. Gerson O. Ludwig, Instituto Nacional de Pesquisas, Brazil

Dr. P.H. Sakanaka, Instituto Fisica, Brazil

The Librarian, Culham Laboratory, England

Mrs. S.A. Hutchinson, JET Library, England

Professor M.N. Bussac, Ecole Polytechnique, France

Librarian, Max-Planck-Institut für Plasmaphysik, Germany

Jolan Moldvai, Reports Library, MTA KFKI-ATKI, Hungary

Dr. P. Kaw, Institute for Plasma Research, India

Ms. P.J. Pathak, Librarian, Insitute for Plasma Research, India

Ms. Clelia De Palo, Associazione EURATOM-ENEA, Italy

Dr. G. Grosso, Instituto di Fisica del Plasma, Italy

Librarian, Naka Fusion Research Establishment, JAERI, Japan

Library, Plasma Physics Laboratory, Kyoto University, Japan

Research Information Center, National Institute for Fusion Science, Japan

Dr. O. Mitarai, Kyushu Tokai University, Japan

Dr. Jiangang Li, Institute of Plasma Physics, Chinese Academy of Sciences, People's Republic of China

Professor Yuping Huo, School of Physical Science and Technology, People's Republic of China

Library, Academia Sinica, Institute of Plasma Physics, People's Republic of China

Librarian, Institute of Physics, Chinese Academy of Sciences, People's Republic of China

Dr. S. Mirnov, TRINITI, Troitsk, Russian Federation, Russia

Dr. V.S. Strelkov, Kurchatov Institute, Russian Federation, Russia

Professor Peter Lukac, Katedra Fyziky Plazmy MFF UK, Mlynska dolina F-2, Komenskeho Univerzita, SK-842 15 Bratislava, Slovakia

Dr. G.S. Lee, Korea Basic Science Institute, South Korea

Institute for Plasma Research, University of Maryland, USA

Librarian, Fusion Energy Division, Oak Ridge National Laboratory, USA

Librarian, Institute of Fusion Studies, University of Texas, USA

Librarian, Magnetic Fusion Program, Lawrence Livermore National Laboratory, USA

Library, General Atomics, USA

Plasma Physics Group, Fusion Energy Research Program, University of California at San Diego, USA

Plasma Physics Library, Columbia University, USA

Alkesh Punjabi, Center for Fusion Research and Training, Hampton University, USA

Dr. W.M. Stacey, Fusion Research Center, Georgia Institute of Technology, USA

Dr. John Willis, U.S. Department of Energy, Office of Fusion Energy Sciences, USA

Mr. Paul H. Wright, Indianapolis, Indiana, USA 
The Princeton Plasma Physics Laboratory is operated by Princeton University under contract with the U.S. Department of Energy.

\author{
Information Services \\ Princeton Plasma Physics Laboratory \\ P.O. Box 451 \\ Princeton, NJ 08543
}

Phone: 609-243-2750

Fax: 609-243-2751

e-mail: pppl_info@pppl.gov

Internet Address: http://www.pppl.gov 